\title{
Fully compliant spatial four-bar mechanism
}

\author{
Engin TANIK* and Volkan PARLAKTAŞ* \\ *Department of Mechanical Engineering, Hacettepe University, \\ Beytepe, Ankara 06800, Turkey \\ E-mail: volkan@hacettepe.edu.tr
}

Received 2 September 2014

\begin{abstract}
This paper presents a novel study on the analysis of the "fully compliant" spatial four-bar mechanism. To the best of our knowledge, any research on "fully compliant" spatial four-bar mechanism is not available in the literature. In the previous study performed by the authors, a "partially compliant" version of the spatial four-bar mechanism was introduced. There was a rigid spherical joint in that case, thus there was no torsional loading at flexural hinges. For the fully compliant case, there is no spherical joint in the structure of the mechanism, thereby there is also torsion available at multiple axis flexural hinges. Design of this fully compliant mechanism is different from the partially compliant case. In this study, deflections of the multiple axis flexural hinges are determined separately as bending and twist. Essential angles for manufacturing a mechanism are determined. A prototype is built and results of the mathematical model are verified with experiments. Finally, a fatigue test is performed. After one and a half million cycles it is observed that there is no indication of any failure. Since there are many applications of rigid spatial four bar mechanisms, it is strongly believed that a fully compliant version of such a mechanism may also find applications.
\end{abstract}

Key words : Spatial four- bar mechanisms, Compliant mechanisms

\section{Introduction}

Four-bar linkages are one of the most commonly used mechanisms in the industry; therefore research on this topic is still very attractive. Recent studies are generally about path generation (Russell and Shen, 2011), (Al-Smadi, et al., 2008), optimal design (Wang, et al., 2013), or geared linkages (Parlaktaş, et al., 2010). Special cases of four-bar linkages are used to obtain mechanical input-output relationship between spatially oriented axes of movement. Oscillatory motion of cranks in 3-dimensional space is generally achieved by spherical (Zhang, 2014) or spatial (Russell, et al., 2009) four-bar mechanisms. An RSSR (revolute-spherical-spherical-revolute) mechanism has two revolute joints of arbitrary orientation attached to the fixed link and two spherical joints connecting the cranks to the coupler link (Söylemez, 1993).

Mechanisms that gain some or all of their motion through the deflection of flexible members are classified as compliant mechanisms. Compliant mechanisms have been investigated due to their significant advantages such as low cost, reduced number of parts, reduced weight, no need for lubrication, less wear, clearance, and noise. Another advantage of compliant mechanisms is that they store elastic energy as they deform, so there is no need for springs to return the mechanism to its original position. However, there are some disadvantages of compliant mechanisms. Fatigue analysis is typically a more vital issue for compliant mechanisms than for their rigid-body counterparts. The motion from the deflection of compliant links is also limited by the strength of the deflecting members. Compliant links that remain under stress for long periods of time or at high temperatures may experience stress relaxation or creep (Howell, 2001). PRBM (pseudo-rigid body model) technique replaces the flexible segments with an equivalent system of rigid links, joints and torsional springs (Howell and Midha, 1996). PRBM can be used for design of compliant mechanisms when the compliant mechanism behavior is such that links can be assumed to be rigid and the flexural pivots can be assumed to behave as torsional springs. Purpose of the PRBM is to provide a simple method of analyzing 
systems that undergo large, nonlinear deflections. There are many studies about planar compliant mechanisms in the literature. Transmission angle in compliant slider-crank mechanism (Tanık, 2011) is an interesting topic. Variable stroke (Tanık and Söylemez, 2011), and uncertainty of applied loads (Kogiso, et al., 2008) are important research areas in compliant mechanisms. Studies on spatial compliant mechanisms are limited in the literature. Constant force mechanisms can be designed as spatial compliant mechanisms (Parlaktaş, 2013). A spatial slider-crank mechanism can be partially compliant or single piece (Parlaktaş and Tanık, 2011, 2014). A compliant cardan universal joint is also a spatial compliant mechanism (Tanık and Parlaktaş, 2012). In the previous study performed by the authors (Tanık and Parlaktaş, 2011) a partially compliant version of the spatial four-bar mechanism was introduced. Since there was a spherical joint in that case, there was no torsional loading at flexural hinges.

In this study, a fully compliant version of the spatial four-bar mechanism with small length flexural pivots is considered. The mechanism is designed to transmit rotary motion in spatially oriented axes. The rotary motions are limited to compliant segments deflections. Therefore, this constraint in the motion of the links does not allow full rotation. Hence, it is a double rocker mechanism. The input and output of the mechanism are the swinging motion of rigid segments 2 and 4 . If a compliant mechanism possesses no kinematic pairs it is called as fully compliant, which is actually a structure with no rigid links. Fully compliant mechanisms can be produced as single piece and they are advantageous since there is no need for assembly. There is no spherical joint in the fully compliant spatial four-bar mechanism, thus there is generally a torsional loading available at multiple axis flexural hinges. Analysis and design of such a compliant mechanism is extremely hard to perform theoretically. In this paper, deflections of multiple axis flexural hinges are determined as bending and twist. Essential angles for manufacturing the mechanism at the undeflected position are determined. In the design example a prototype is built and it is shown that the results of the proposed theoretical model and the real model are consistent. Finally, a fatigue test is performed.

\section{Kinematic analysis of RSSR linkage}

Before analysis of the compliant RSSR, a kinematic analysis procedure for rigid RSSR is required in order to employ the PRBM. Disregarding the rotation of the coupler about its axis (which is also constrained in the compliant RSSR), the mechanism possesses one degree-of-freedom. A RSSR converts a rotation about an axis into a rotation about another axis in the space. Referring to Fig. 1, $a_{2}=$ length of driving crank (link 2), $a_{3}=$ length of coupler link (link 3), $a_{4}=$ length of driven link (link 4), $p=\mid \mathrm{OCl}$ length of common perpendicular between the axis of turning pairs, $f=|\mathrm{OF}|=$ distance from the foot of the common perpendicular to the center of crank circle described by point $\mathrm{A}$, $g=|\mathrm{CG}|=$ distance from the foot of common perpendicular to the center of crank circle described by point B, $\theta=$ crank angle measured counter-clockwise (in the right handed sense about $\mid \mathrm{OFI}$ ) from positive $\mathrm{X}$-axis, $\xi=$ angular offset; angle between axis of turning pairs measured according to the right hand rule from the output axis to the input axis (about the positive $\mathrm{X}$-axis), $\chi=$ angular displacement of driven link measured counter clockwise (in the right handed sense about CG) from the positive $\mathrm{X}$-axis. The relationship between input $\theta$ and output $\chi$ can be obtained by substituting trigonometric identities into general displacement equation (Söylemez and Freudenstein, 1982).

$\chi=2 \tan ^{-1}\left(\frac{C \pm \sqrt{C^{2}-A^{2}+B^{2}}}{A+B}\right)$

where, $\quad A=p_{1}-p_{2} \cos \theta-p_{5} \sin \theta$

$B=p_{4} \cos \theta-p_{3}, \quad C=p_{7} \sin \theta-p_{6}$

$p_{1}=p^{2}+a_{2}^{2}-a_{3}^{2}+a_{4}^{2}+f^{2}+g^{2}-2 f g \cos \xi, \quad p_{2}=2 p a_{2}, \quad p_{3}=2 p a_{4}, \quad p_{4}=2 a_{2} a_{4}, \quad p_{5}=2 g a_{2} \sin \xi, \quad p_{6}=2 f a_{4} \sin \xi$,

$p_{7}=2 a_{2} a_{4} \cos \xi$

The unit vectors on links 2, 3 and 4 are determined as:

$$
\begin{aligned}
\overrightarrow{e_{a 2}} & =\cos \theta \overrightarrow{\mathrm{\imath}}+\sin \theta \overrightarrow{\mathrm{j}} \\
\overrightarrow{e_{a 3}} & =\frac{p+a_{4} \cos \chi-a_{2} \cos \theta}{a_{3}} \overrightarrow{\mathrm{\imath}}+\frac{g \sin \xi+a_{4} \sin \chi \cos \xi-a_{2} \sin \theta}{a_{3}} \overrightarrow{\mathrm{\jmath}}+\frac{g \cos \xi-a_{4} \sin \chi \sin \xi-f}{a_{3}} \overrightarrow{\mathrm{k}} \\
\overrightarrow{e_{a 4}} & =\cos \chi \overrightarrow{\mathrm{\imath}}+\sin \chi \cos \xi \overrightarrow{\mathrm{\jmath}}-\sin \chi \sin \xi \overrightarrow{\mathrm{k}}
\end{aligned}
$$




\section{Fully compliant spatial four-bar mechanism}

The fully compliant spatial four-bar mechanism in the most general form is presented in Fig. 1a. The PRBM of this compliant mechanism is shown in Fig. 1b. PRBM is accurate even for large deflections for many cases. If bending is the dominant loading in a flexural hinge, PRBM is more accurate. The nature of small-length flexural pivots ensures that the assumption that bending is the predominant loading is accurate in most applications. PRBM is useful in the early design phases where many design iterations are required to design a mechanism that fulfills the design objectives specified. It is usually preferred to employ PRBM to make initial approximations in the design to obtain a general understanding of mechanism characteristics and then use other methods to improve the design (Howell, 2001).

For the fully compliant spatial four-bar mechanism, the deflection of the multiple-axis flexural hinges contains both twist and bending. However, torsion at the single-axis flexural hinges can be neglected if height of cross section is large enough. This situation was verified by the authors in their previous papers which are cited in Sec.1. Deflection of flexural hinges is one of the most important design criteria in compliant mechanisms, because stresses occurred are proportional to deflections. However, for the spatial flexural hinges determination of deflection is very complex. Deflections of the multiple-axis flexural hinges are determined separately as bending and twist in the next sections.

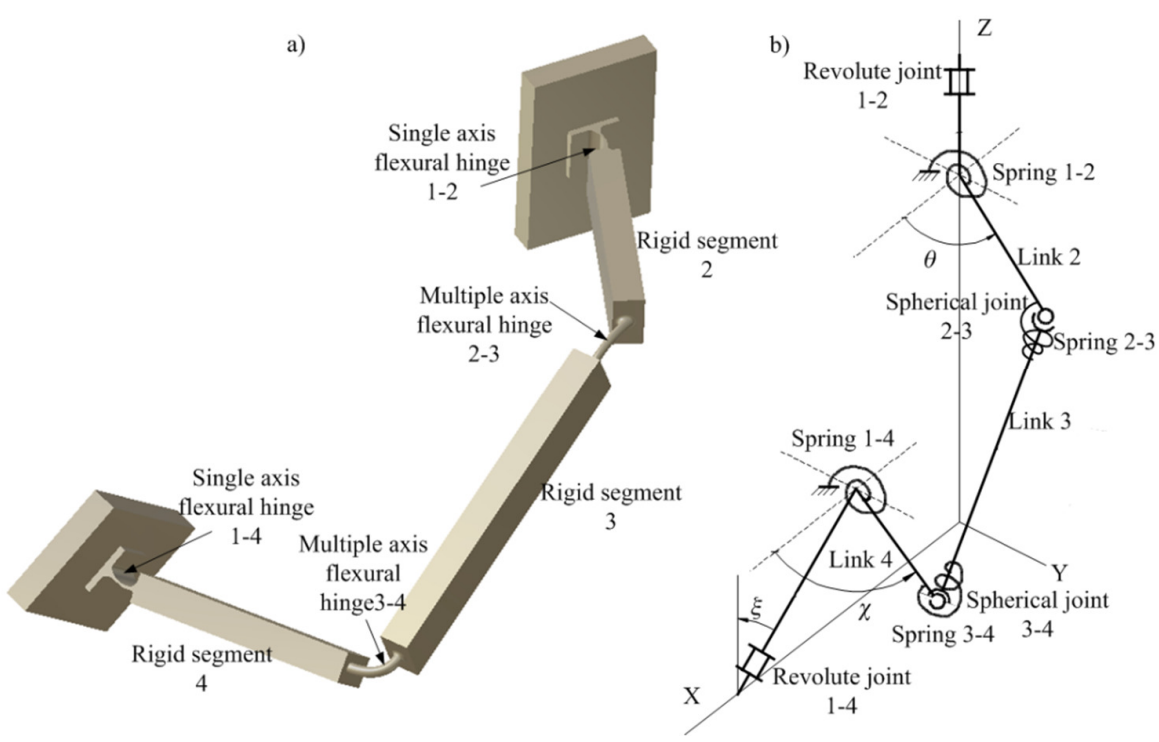

Fig. 1. Compliant RSSR mechanism and its PRBM

\section{Determination of bending of the flexural hinges}

In this section, initially bending of the multiple-axis flexural hinges will be determined by using the method which was proposed in the previous work (Tanık and Parlaktaş, 2011). It was verified that if the instantaneous compliant axis is determined from the undeflected and the current positions, then the case can be considered as an initially straight flexural beam subjected to a single plane bending along its new compliant axis (Parlaktaş and Tanık, 2011). At the end of this section deflection of the single-axis flexural hinges will be determined with respect to crank position.

In Fig. 2 the mechanism is shown at the current and undeflected positions. Here, the current position is for an arbitrary crank position and the mechanism is assembled to the ground. The undeflected position occurs when the single-axis flexural hinge 1-2 is disassembled from the ground. The unit vectors on the compliant axis of multiple axis flexural hinge 3-4, on the undeflected and current positions of rigid segment 3 are $\overrightarrow{e_{\alpha 1}}, \overrightarrow{e_{\mathrm{u} 1}}$ and $\overrightarrow{e_{\mathrm{a} 3}}$ respectively as shown in Fig. 2. $\alpha_{1}$ is the angle between the undeflected and current positions. The essential angles for manufacturing are $\mu$ and $\varphi$. (Note that $\mu$ and $\varphi$ are design parameters that define the manufacturing (or undeflected) position of multi-axis flexural hinge 3-4). All of these parameters were determined in the previous study (Tanık and Parlaktaş, 2011).

In this paper bending of the multiple-axis flexural hinge 2-3 is determined similarly. In Fig. 3 the mechanism is shown at the current and undeflected positions. Here, the current position is for arbitrary crank position and the 
mechanism is assembled to the ground. The undeflected position occurs when the single-axis flexural hinge 1-4 is disassembled from ground. The unit vectors on the compliant axis of the multiple axis flexural hinge 2-3, on the undeflected and current positions of rigid segment 3 are $\overrightarrow{e_{\alpha 2}}, \overrightarrow{e_{\mathrm{u} 2}}$ and $\overrightarrow{e_{\mathrm{a} 3}}$ respectively as shown in Fig. 3. Here $\alpha_{2}$ is the angle between the undeflected and current positions and it can be determined as:

$\alpha_{2}=\cos ^{-1}\left(\left[\overrightarrow{e_{u 2}}\right]^{\mathrm{T}}\left[\overrightarrow{e_{a 3}}\right]\right)$

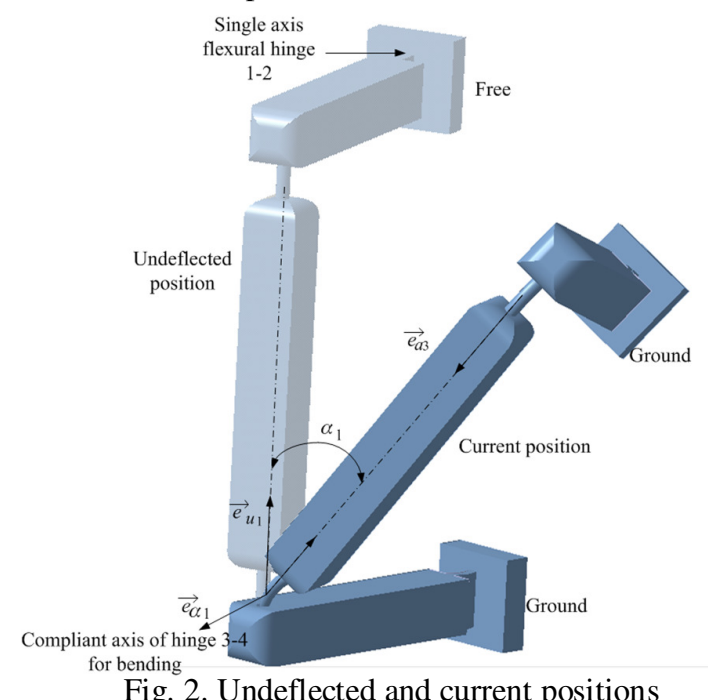

Fig. 2. Undeflected and current positions

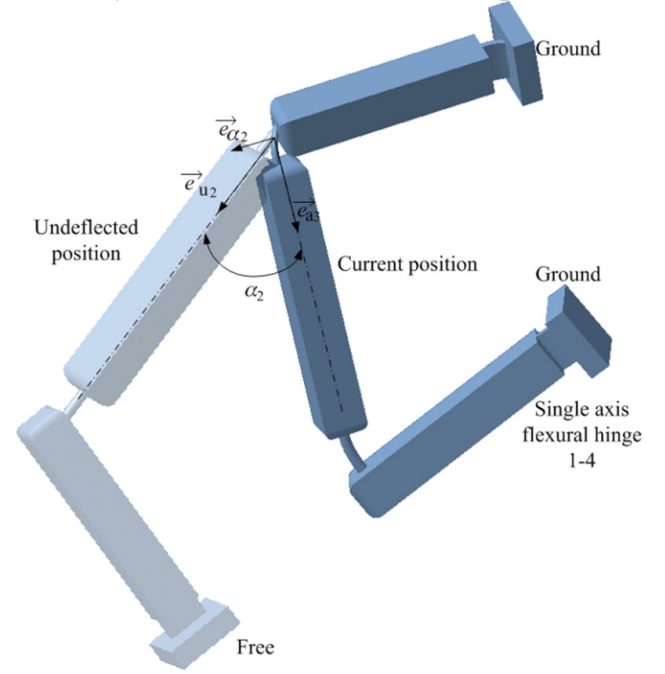

Fig. 3. Undeflected and current positions

In order to obtain $\overrightarrow{e_{\mathrm{u} 2}}$ the following procedure can be employed; let a unit vector $-\overrightarrow{\mathrm{k}}$ is located on rigid segment $-2(-\overrightarrow{\mathrm{k}}$ is parallel to Z-axis) where $\theta$ is equal to zero (Fig. 4). To transform this unit vector along the direction of the undeflected position rigid segment-3, initially unit vector $\overrightarrow{-\mathrm{k}}$ is rotated about X-axis ccw by an angle $\gamma$, then about Y-axis ccw by an angle $\psi$, by using Euler rotations Eq. (6). (Note that $\gamma$, and $\psi$ are structure parameters that defines the undeflected position of multi-axis flexural hinge 2-3 and their limits are: $-\pi \leq \mu \leq \pi$ and $0 \leq \varphi \leq 2 \pi$ ).
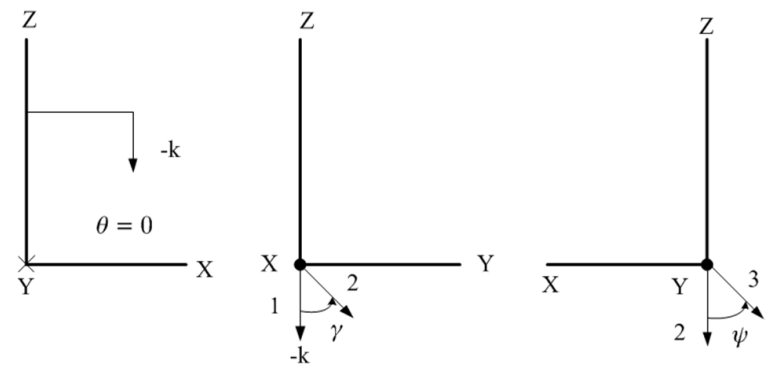

Fig. 4. Obtaining the undeflected position of multi-axis flexural hinge 2-3 
Hence $\left[A_{2}\right]$ is determined as:

$$
\begin{aligned}
& {\left[A_{2}\right]=\left[\begin{array}{ccc}
\cos \psi & 0 & \sin \psi \\
0 & 1 & 0 \\
-\sin \psi & 0 & \cos \psi
\end{array}\right]\left[\begin{array}{ccc}
1 & 0 & 0 \\
0 & \cos \gamma & -\sin \gamma \\
0 & \sin \gamma & \cos \gamma
\end{array}\right]\left[\begin{array}{c}
0 \\
0 \\
-1
\end{array}\right]} \\
& {\left[A_{2}\right]=\left[\begin{array}{c}
-\sin \psi \cos \gamma \\
\sin \gamma \\
-\cos \psi \cos \gamma
\end{array}\right]}
\end{aligned}
$$

Then $\left[A_{2}\right]$ is rotated about z-axis ccw by an angle $\theta$.

$$
\left[B_{2}\right]=\left[\begin{array}{ccc}
\cos \theta & -\sin \theta & 0 \\
\sin \theta & \cos \theta & 0 \\
0 & 0 & 1
\end{array}\right]\left[A_{2}\right]
$$

$\left[\overrightarrow{e_{\mathrm{u} 2}}\right]$ is equal to $\left[B_{2}\right]$ for any crank position.

$$
\left[\overrightarrow{e_{u 2}}\right]=\left[B_{2}\right]=\left[\begin{array}{c}
-\cos \theta \sin \psi \cos \gamma-\sin \theta \sin \gamma \\
-\sin \theta \sin \psi \cos \gamma+\cos \theta \sin \gamma \\
-\cos \psi \cos \gamma
\end{array}\right]
$$

Now $\left[\overrightarrow{e_{u 2}}\right]$ is known, thus $\alpha_{2}$ can be determined from Eq. (5), and $\overrightarrow{e_{\alpha 2}}$ can be determined as:

$\overrightarrow{e_{\alpha 2}}=\frac{\overrightarrow{e_{u 2}} \times \overrightarrow{e_{a 3}}}{\left|\overrightarrow{e_{u 2}} \times \overrightarrow{e_{a 3}}\right|}$

Deflections of the single-axis flexural hinges are determined with respect to crank angle as,

$\theta_{T}=\left(\theta-c_{12}\right)$

$\chi_{T}=\left(\chi-c_{14}\right)$

Here, $c_{i j}$ are the initial angle between the $i$ th and $j$ th segments.

\section{Determination twist of the flexural hinges}

In this section, twist of the multiple-axis flexural hinges will be determined by using a novel procedure. For this purpose the mechanism and its PRBM without springs (red line) are shown at the current and undeflected positions again in Fig. 5 and new parameters are introduced. At the undeflected position, there is no deformation at the flexural hinges 2-3, 3-4 and 1-4 (it does not matter if there is deformation at single axis flexural hinges).

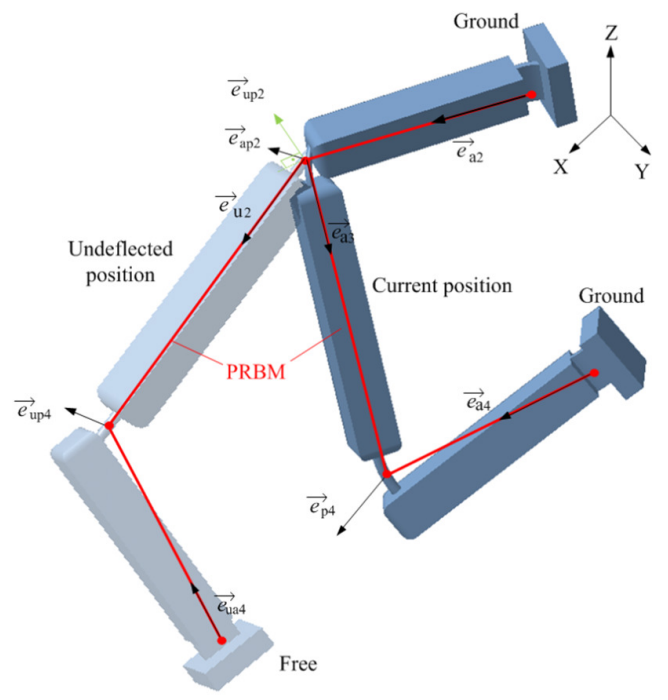

Fig. 5. Undeflected and current positions 
When the mechanism is assembled to the ground and an input motion is given, rotational differences occur between the cranks (rigid segments-2 and 4) except some special cases (e.g. parallelogram four-bar). Due to this difference, rigid segment-3 must essentially rotate about its own axis. Therefore, a "twist" occurs at the multiple-axis flexural hinges. The amount of total twist angle is shared by the flexural hinges according to their geometry.

The total twist can be determined as follows: Unit vectors on rigid segments-2 and on the undeflected position of rigid segment 3 are $\left(\overrightarrow{e_{\mathrm{a} 2}}\right.$ and $\overrightarrow{e_{\mathrm{u} 2}}$ ) obtained in previous sections. Initially $\overrightarrow{e_{\mathrm{a} 2}}$ is rotated $90^{\circ} \mathrm{ccw}$ about $\overrightarrow{e_{\mathrm{u} 2}}$ and the unit vector $\overrightarrow{e_{\mathrm{ap} 2}}$ is determined. Mathematically;

$\left[\overrightarrow{e_{a p 2}}\right]=[R]\left[\overrightarrow{e_{a 2}}\right]$

$[R]=$

$\left[\begin{array}{ccc}\cos (\pi / 2)+e_{u 2 x}{ }^{2}(1-\cos (\pi / 2)) & e_{u 2 x} e_{u 2 y}(1-\cos (\pi / 2))-e_{u 2 z} \sin (\pi / 2) & e_{u 2 x} e_{u 2 z}(1-\cos (\pi / 2))+e_{u 2 y} \sin (\pi / 2) \\ e_{u 2 x} e_{u 2 y}(1-\cos (\pi / 2))+e_{u 2 z} \sin (\pi / 2) & \cos (\pi / 2)+e_{u 2 y}^{2}(1-\cos (\pi / 2)) & e_{u 2 z} e_{u 2 y}(1-\cos (\pi / 2))-e_{u 2 x} \sin (\pi / 2) \\ e_{u 2 x} e_{u 2 z}(1-\cos (\pi / 2))-e_{u 2 y} \sin (\pi / 2) & e_{u 2 z} e_{u 2 y}(1-\cos (\pi / 2))+e_{u 2 x} \sin (\pi / 2) & \cos (\pi / 2)+e_{u 2 z}{ }^{2}(1-\cos (\pi / 2))\end{array}\right]$

Let a unit vector $\overrightarrow{e_{\mathrm{up} 2}}$ be perpendicular to $\overrightarrow{e_{\mathrm{u} 2}}$ and $\overrightarrow{e_{\mathrm{ap} 2}}\left(\overrightarrow{e_{\mathrm{up} 2}}\right.$ is perpendicular to the plane which is formed by $\overrightarrow{e_{\mathrm{u} 2}}$ and $\overrightarrow{e_{\mathrm{ap} 2}}$. The exact destination of $\overrightarrow{e_{\mathrm{up} 2}}$ is the center of spherical joint 2-3 which is assumed to be on the PRBM as seen in Figures 5 and 6. Hence, $\overrightarrow{e_{\mathrm{up} 2}}$ can be obtained as:

$\left[\overrightarrow{e_{u p 2}}\right]=\frac{\left[\overrightarrow{e_{a p 2}}\right] x\left[\overrightarrow{e_{u 2}}\right]}{\left|\overrightarrow{e_{a p 2}} \times \overrightarrow{e_{u 2}}\right|}$

The unit vectors $\overrightarrow{e_{u p 2}}$ and $\overrightarrow{e_{u 2}}$ constitutes a plane as shown in Fig. 6 .

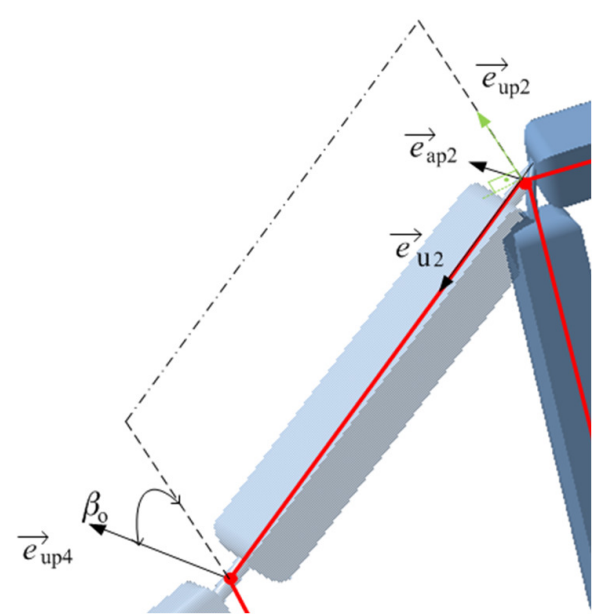

Fig. 6. The plane constituted by unit vectors $\overrightarrow{e_{\mathrm{up} 2}}$ and $\overrightarrow{e_{\mathrm{u} 2}}$

If $\overrightarrow{e_{\mathrm{up} 2}}$ is rotated about $\overrightarrow{e_{\mathrm{u} 2}}$ by an angle $\beta_{0} \mathrm{ccw}$, another unit vector $\overrightarrow{e_{\mathrm{up} 4}}$ is determined. It should be noted that $\beta_{0}$ is a newly introduced structure angle. $\overrightarrow{e_{\mathrm{up} 4}}$ is at the center of the spherical joint 3-4 of the PRBM as seen in Fig. 6, and it is determined as:

$\left[\overrightarrow{e_{u p 4}}\right]=[R]\left[\overrightarrow{e_{u p 2}}\right]$

$[R]=\left[\begin{array}{ccc}\cos \beta_{0}+e_{u 2 x^{2}}\left(1-\cos \beta_{0}\right) & e_{u 2 x} e_{u 2 y}\left(1-\cos \beta_{0}\right)-e_{u 2 z} \sin \beta_{0} & e_{u 2 x} e_{u 2 z}\left(1-\cos \beta_{0}\right)+e_{u 2 y} \sin \beta_{0} \\ e_{u 2 x} e_{u 2 y}\left(1-\cos \beta_{0}\right)+e_{u 2 z} \sin \beta_{0} & \cos \beta_{0}+e_{u 2 y}^{2}\left(1-\cos \beta_{0}\right) & e_{u 2 z} e_{u 2 y}\left(1-\cos \beta_{0}\right)-e_{u 2 x} \sin \beta_{0} \\ e_{u 2 x} e_{u 2 z}\left(1-\cos \beta_{0}\right)-e_{u 2 y} \sin \beta_{0} & e_{u 2 z} e_{u 2 y}\left(1-\cos \beta_{0}\right)+e_{u 2 x} \sin \beta_{0} & \cos \beta_{0}+e_{u 2 z}{ }^{2}\left(1-\cos \beta_{0}\right)\end{array}\right]$

The unit vector $\overrightarrow{e_{\mathrm{up} 4}}$ is on the plane which is formed by $\overrightarrow{e_{\mathrm{u} 2}}$ and $\overrightarrow{e_{\mathrm{ua} 4}}$ (undeflected positions of rigid segments 3 and 4). Hence, the relative position of rigid segment-4 with respect to rigid segment- 2 is designated with the angle $\beta_{0}$. At this stage essential angles which are necessary to produce the mechanism are determined.

The unit vector on rigid segment-4, $\overrightarrow{e_{\mathrm{a} 4}}$ is a known parameter (Eq. 4). The plane formed by the unit vectors $\overrightarrow{e_{u p 2}}$ 
and $\overrightarrow{e_{\mathrm{a} 3}}$ is shown in Fig. 7. The angle between $\overrightarrow{e_{\mathrm{a} 4}}$ and the plane which is formed by $\overrightarrow{e_{\mathrm{up} 2}}$ and $\overrightarrow{e_{\mathrm{a} 3}}$ is $\beta$. This is the angle difference of rigid segments 2 and 4 for an arbitrary position of the mechanism disregarding bending. Because any rotation of cranks that causes a bending cannot change angle $\beta$. Thus, $\beta$ can also be called as the total angle of twist of both multi-axis flexural hinges.

To determine $\beta$, initially a unit vector which is perpendicular to the plane formed by $\overrightarrow{e_{\mathrm{up} 2}}$ and $\overrightarrow{e_{\mathrm{a} 3}}$ is obtained. The angle between this perpendicular vector and $\overrightarrow{e_{\mathrm{a}}}$ is determined by dot product. The difference of this angle from $90^{\circ}$ is the angle of twist, as given in Eq. 15 .

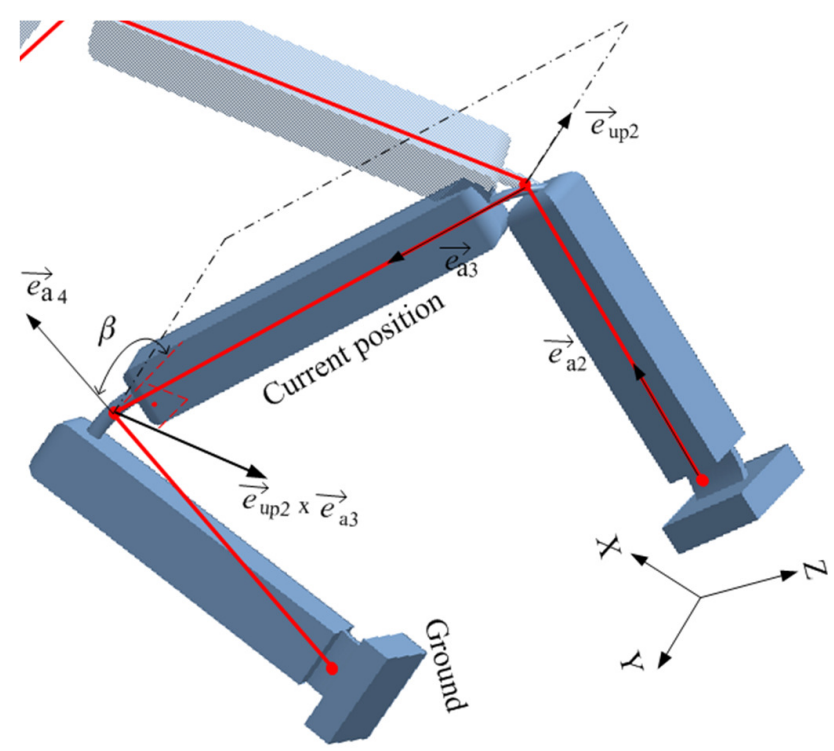

Fig. 7. The plane constituted by unit vectors $\overrightarrow{e_{\mathrm{up} 2}}$ and $\overrightarrow{e_{\mathrm{a} 3}}$

$\beta=\cos ^{-1}\left\{\frac{\overrightarrow{e_{a 4}}\left(\overrightarrow{e_{u p 2}} \times \overrightarrow{e_{a 3}}\right)}{\left|\overrightarrow{e_{u p 2}} \times \overrightarrow{e_{a 3}}\right|}\right\}-\frac{\pi}{2}$

Since angle $\beta_{0}$ is the initial value of $\beta$, the total angle of twist is:

$\beta_{T}=\beta-\beta_{0}$

\section{Example}

The purpose of this example is to compare the results of the proposed theoretical model with FEA and a real model. The link lengths of the PRBM are determined by heuristic optimization according to some design considerations which are listed as follows:

The stresses at the flexural hinges will be high if deflections of the hinges are also high. Hence, low deflection values are aimed. On the other hand, to obtain a useful mechanism in practice the output stroke should not be low. For the easiness of production a mechanism which is on $\mathrm{x}-\mathrm{z}$ plane while at rest (undeflected) is designed. Let the link proportions of the PRBM of the compliant mechanism be; $p=100 \mathrm{~mm}, f=100 \mathrm{~mm}, g=0, a_{2}=100 \mathrm{~mm}, a_{3}=73.5 \mathrm{~mm}, a_{4}$ $=32.5 \mathrm{~mm}$, and $\xi=90^{\circ}$. The structure angles are optimization parameters which are explained as in Sec.4. Let these angles be $\mu=137.7^{\circ}, \varphi=-90^{\circ}, \gamma=0, \psi=-12.6^{\circ}$. Last structure parameter angle $\beta_{0}$ is equal to zero since the compliant hinges are undeflected at the $\mathrm{x}-\mathrm{z}$ plane. For the same reason the initial angles of the single-axis flexural hinges are also zero $\left(c_{12}=c_{14}=0\right)$.

By using the analysis procedure given in this study, bending and total twist of the flexural hinges are displayed in Fig. 8. The maximum bending values are below $16^{\circ}$. The total twist of multi-axis flexural hinges is displayed with purple, and its maximum value is $16.5^{\circ}$.

Finally the hinges are dimensioned considering the related literature. Although there is no strict method to design length of the compliant segments of such mechanisms, there are some recommendations available in compliant 
mechanisms literature: Ratio of length of the compliant segment to neighbor rigid segment should be smaller than 1/10 in most applications when PRBM method is implemented. (Howell, 2001) If this ratio is larger than $\sim 1 / 10$ generally the error involved between PRBM and real model will most probably be in unacceptable range. After the length of the joint is determined, then the minimum thickness of the joint is decided. As a rule of thumb the smaller cross section yields smaller stress for the same deflection. However, we cannot conclude that thinner compliant segment is always better, because the smaller joint thickness decreases load carrying capacity of a compliant mechanism. Also, if a specific stiffness (spring constant) is required the length and diameter of the joint must be considered simultaneously.

Let the length of the multi-axis flexural hinges are $9 \mathrm{~mm}$ and diameter of the cross section is $1.5 \mathrm{~mm}$. Let the length of the single-axis flexural hinge 1-2 is $7 \mathrm{~mm}$ and the length of the single-axis flexural hinge 1-4 is $4.5 \mathrm{~mm}$. Let the thickness and height of the single-axis flexural hinges are $1.5 \mathrm{~mm}$ and $10 \mathrm{~mm}$ respectively. Here lengths of the flexural hinges are the effective lengths. There are fillets at each ends of the hinges to eliminate stress concentrations, which are not counted in the length. Also note that the material of the mechanism is selected as polypropylene $(\mathrm{E}=1.3$ $\mathrm{GPa})$.

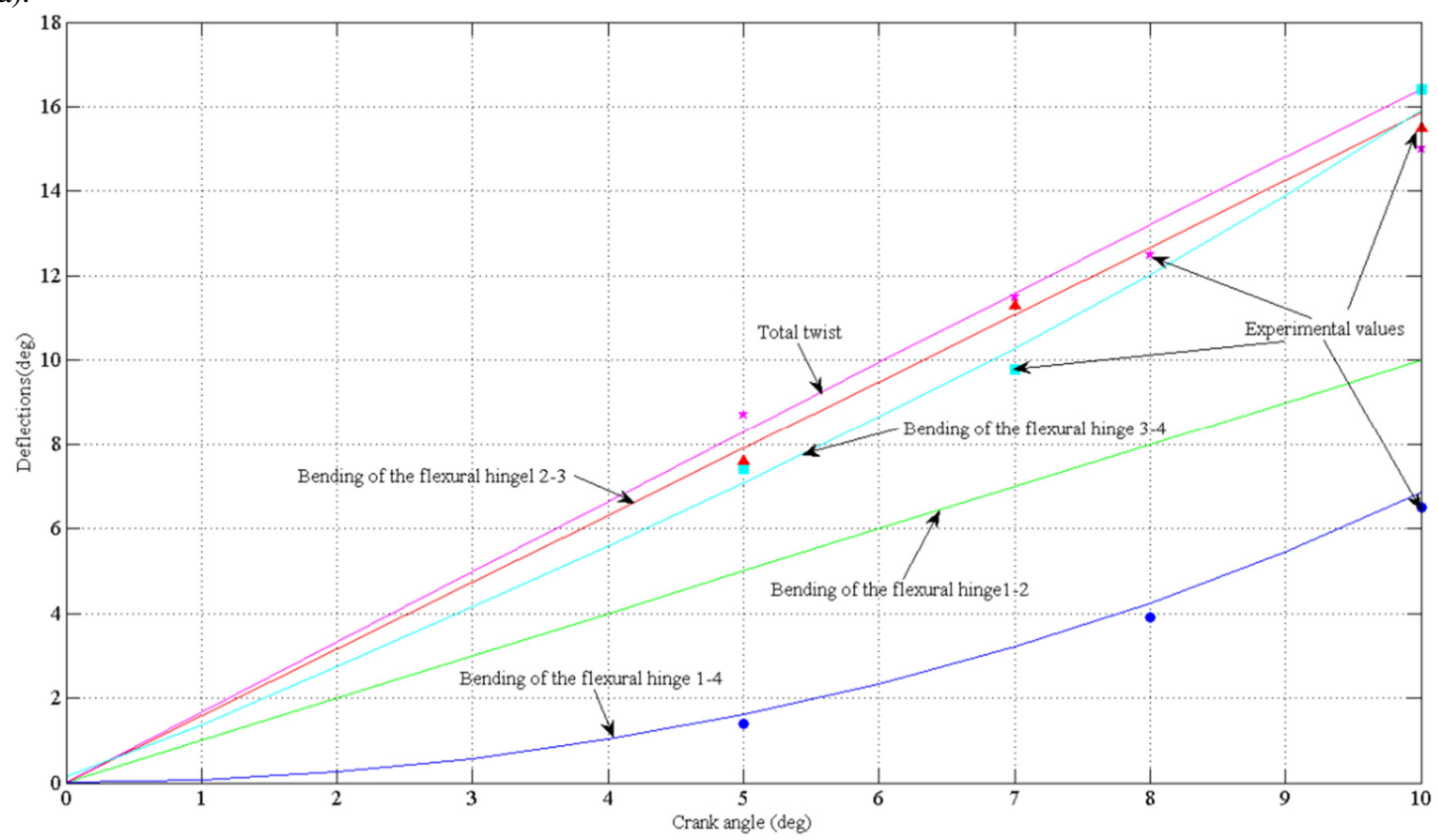

Fig. 8. Input/output relationship

After the design stage, the mechanism is analyzed by a FEA software. A nonlinear analysis module is selected since the deflections are high. The elements are hex type. The input crank is rotated $10^{\circ}$ about z-axis and $6.9^{\circ}$ rotation of output crank (equal to the deflection of single axis flexural hinge 1-4) is obtained where $7^{\circ}$ is obtained in the analytical model. Maximum stresses occur at the multi-axis flexural hinges as expected, because there is twist and bending available together. Von-Mises stresses at flexural hinges are displayed in Fig. 9. The maximum stress is at the multi-axis flexural hinge 2-3 with a value of 35.8 MPa. These are static stresses and do not correlate with fatigue strength. The material of the mechanism is selected as polypropylene and the fatigue properties of polymers are not well defined like steels in the literature (Howell, 2001). Therefore, it is not feasible to calculate the fatigue life of a compliant mechanism made of polypropylene by FEA software.

A real model of the mechanism is manufactured with the given dimensions. In order to illustrate the force transmission in spatial axis, rigid segment-4 is produced with an extension and a switch is assembled to the ground (Fig. 10). Hence, when an input motion is given to the crank, rigid segment-4 opens the switch. The related e-component is given in the Appendix. 


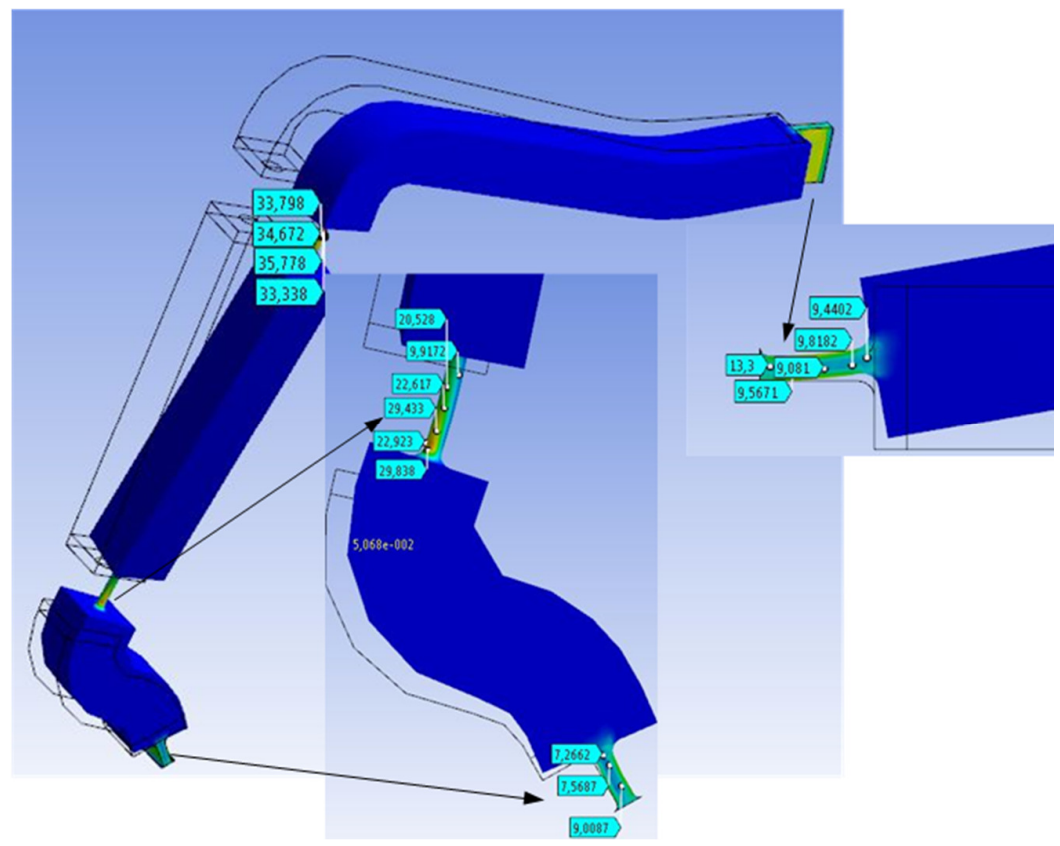

Fig. 9. Von-Mises stresses at flexural hinges

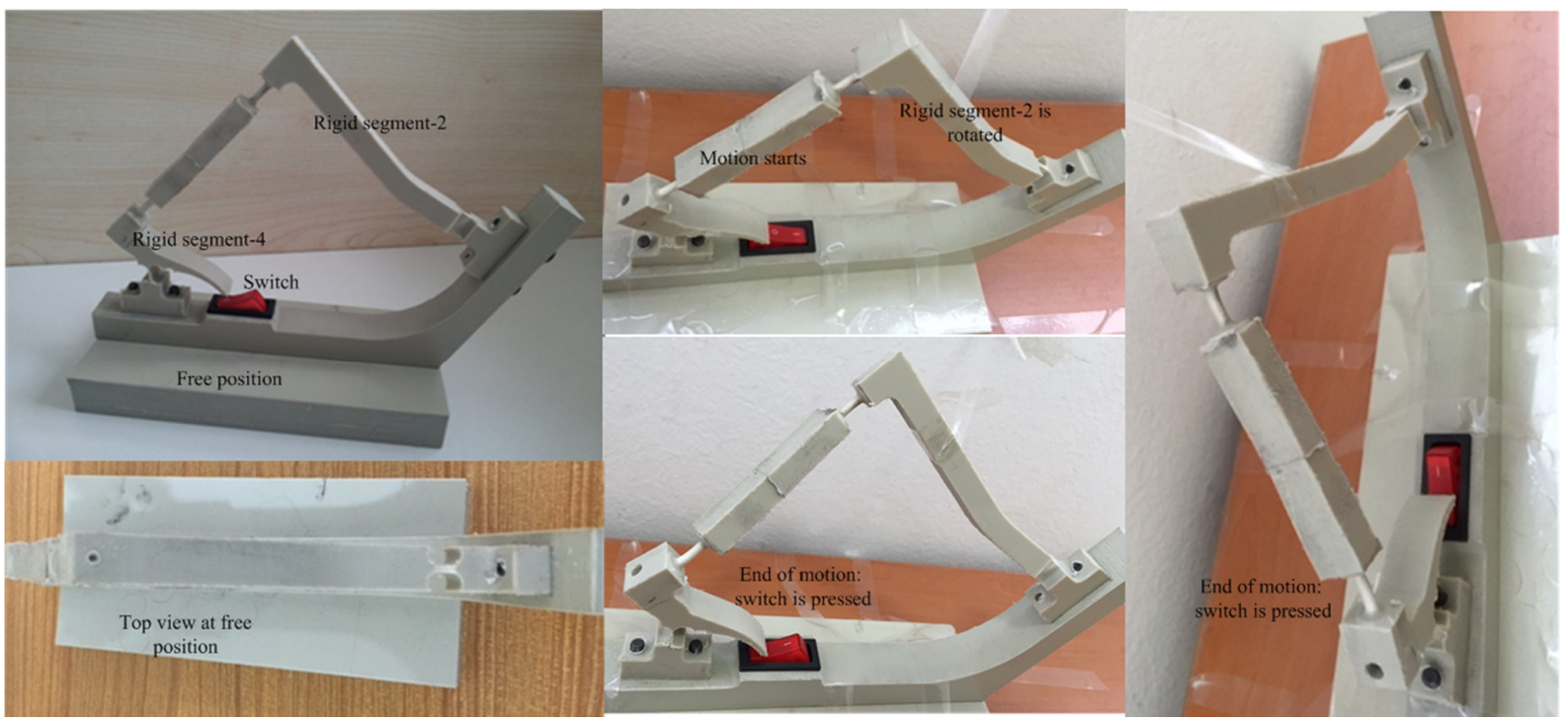

Fig. 10. Prototype\#1

For obtaining experimental deflection values, another prototype of the same mechanism is manufactured in $\mathrm{x} 2$ scale except thickness and diameter of the hinges. The second model is scanned with an optic CMM (coordinate measuring machine) when the crank is fixed to $10^{\circ}$ (Fig. 11). The data from CMM is exported to a solid model software. The position of the mechanism at $10^{\circ}$ is superposed with the undeflected position and necessary angles are measured by the software. Thus, bending and twist of the multi-axis flexural hinges are determined experimentally. The same procedure is repeated for different crank positions and same data are collected. The experimental values are very close to the analytical results as displayed in Fig. 8. 


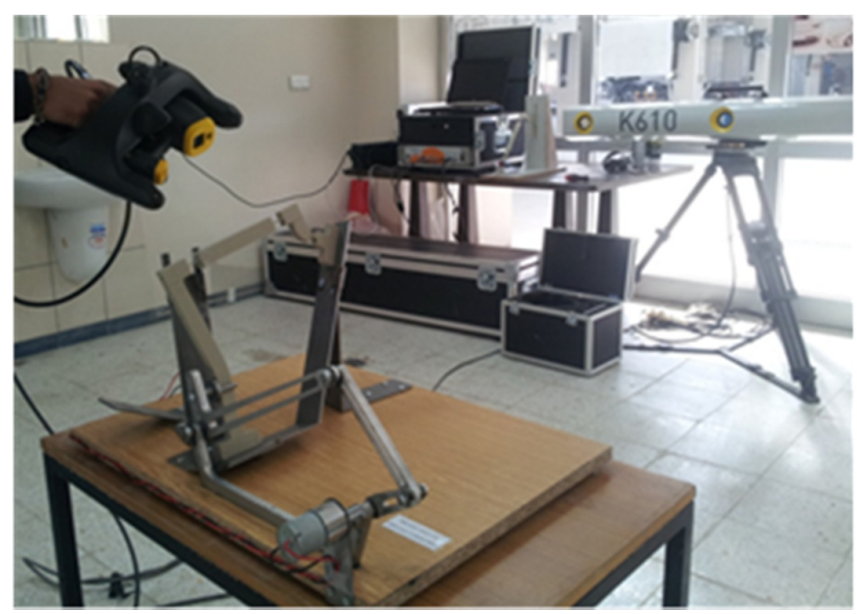

Fig. 11. Scanning Prototype\#2

\section{Experimental fatigue test}

One of the frequently asked questions in compliant mechanisms design is the fatigue life of the compliant hinges. First of all, in machine design it is not an obligation to have an infinite life for all real world applications. Besides, fatigue life of compliant mechanisms is not short if properly designed. To verify this claim, an experimental fatigue test is carried out.

For this purpose, a fatigue test setup is designed and produced as seen in Fig. 12. A good choice for actuator of the compliant mechanism is a variable stroke crank-rocker four-bar mechanism. The output link of the crank-rocker mechanism is assembled to the input link of the compliant mechanism. During this assembly the output link of the rigid mechanism and crank of the compliant mechanisms should have the same rotation axis (Fig. 13). Also, it is important to rotate the crank of the compliant mechanism equally in each direction. Thus, completely reversed loading can be obtained. After mounting an electric motor, sensor, counter and power supply, the experimental setup is completed as seen in Fig. 12.

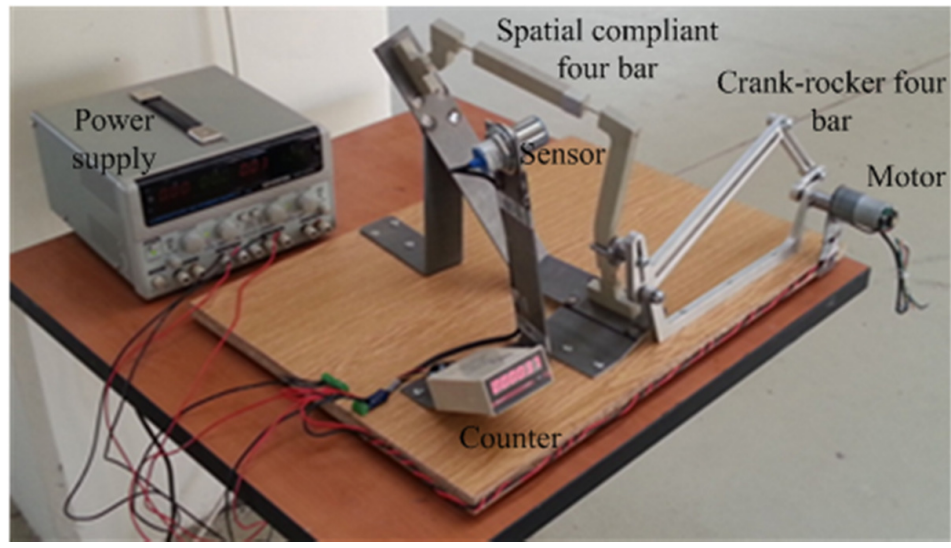

Fig. 12. Fatigue experimental setup

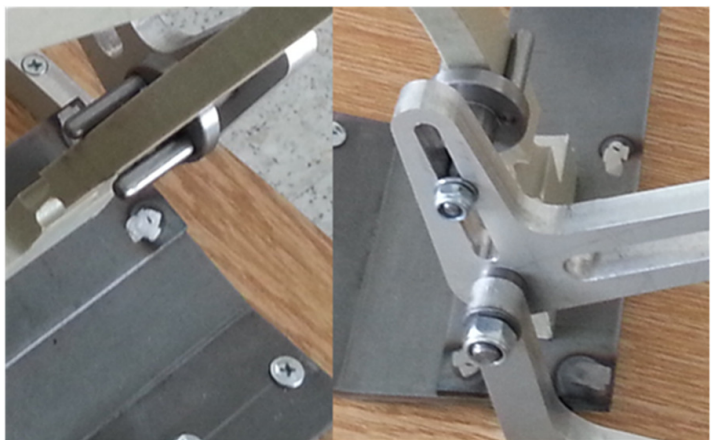

Fig. 13. Assembling the compliant mechanism to fatigue setup 
Considering a safety factor the set-up is arranged so that the crank of the compliant mechanism rotates $8^{\circ}$ in each direction. This rotation leads a maximum stress of $25 \mathrm{MPa}$ in one of the compliant hinges. After the mechanism is operated for 1.5 million cycles, there is no indication of failure at all: There is no rupture, no tear even no color change. The experiment is recorded at different cycles. The related e-component is given in the Appendix.

\section{Conclusions}

This paper presents the first study on a fully compliant spatial four-bar mechanism. A novel analysis approach for a fully compliant spatial four-bar mechanism with small length flexural pivots is presented by using its PRBM.

A method is proposed to determine the deflections of the multiple axis flexural hinges as bending and twist. It should be noted that these calculations are approximate since PRBMs generally yields some small error in analysis and design. It is usually preferred to employ PRBM to make initial approximations in the design to obtain a general understanding of mechanism characteristics, and then perform a FEA to improve the design.

The essential angles for manufacturing the mechanism at the undeflected position are determined. In the design example a real model is built and it is shown that the results of the proposed theoretical model, FEA model and the real model are consistent. Finally, the prototype is exposed to a fatigue test. After one and a half million cycles it is observed that there is no indication of fatigue.

There are numerous applications of rigid spatial four bar mechanisms; however manufacturing an inexpensive and precise rigid RSSR is not generally possible. Fully compliant mechanisms can be produced as single piece and they are very advantageous since there is no need for assembly. A fully compliant version of such this mechanism will reduce the cost considerably. Therefore, it is strongly believed that fully compliant spatial four-bar mechanisms may find many applications.

\section{Appendix. Supplementary data}

http://www.youtube.com/watch?v=Qa_U42SdTAE\&feature=youtu.be (fatigue)

http://www.youtube.com/watch?v=B5TUkA69yH4\&feature=youtu.be (small real model)

\section{Acknowledgements}

The authors thank graduate student Göktuğ Burak Çalık for the FEA. This project is sponsored by Turkish Scientific and Technological Research (TÜBITAK) Center Career Award 111M154 of the corresponding author.

\section{References}

Al-Smadi, Y. M., Russell, K. and Sodhi, R. S., Planar four-bar path generation with static structural conditions, Journal of Advanced Mechanical Design, Systems, and Manufacturing, Vol. 2, No. 5 (2008), pp. 926-936.

Howell, L.L, Compliant Mechanisms (2001), John Wiley and Sons Inc., New York.

Howell, L. L. and Midha, A., Evaluation of equivalent spring stiffness for use in a pseudo-rigid body model of large deflection compliant mechanisms, Transactions of the ASME, Journal of Mechanical Design, Vol. 118 (1996), pp. 126-131.

Kogiso, N., Ahn, W.J., Nishiwaki, S., Izui, K. and Yoshimura, M., Robust topology optimization for compliant mechanisms considering uncertainty of applied loads, Journal of Advanced Mechanical Design, Systems, and Manufacturing, Vol. 2, No. 1 (2008), pp. 96-107.

Parlaktaş, V., Söylemez, E. and Tanık, E., On the synthesis of a geared four-bar mechanism, Mechanism and Machine Theory, Vol. 45, No .8 (2010), pp. 1142-1152.

Parlaktaş, V., Spatial compliant constant-force mechanism, Mechanism and Machine Theory, Vol. 67 (2013), pp. 152-165.

Parlaktaş, V. and Tanık, E., Partially compliant spatial slider-crank (RSSP) mechanism, Mechanism and Machine Theory, Vol. 46 (2011), pp. 1707-1718. 
Parlaktaş, V. and Tanık, E., Single piece compliant spatial slider-crank mechanism, Mechanism and Machine Theory, Vol. 81 (2014), pp. 1-10.

Russell, K. and Shen, J., Planar four-bar motion and path generation with order and branching conditions, Journal of Advanced Mechanical Design, Systems, and Manufacturing, Vol. 5, No. 4 (2011), pp. 264-273.

Russell, K., Shen, Q., Lee, W.T. and Sodhi, R. S., On the synthesis of spatial RRSS motion generators with prescribed coupler loads, Journal of Advanced Mechanical Design, Systems, and Manufacturing, Vol. 3, No. 3 (2009), pp. 236-244.

Söylemez, E., Transmission optimization of right-angled four-bar mechanisms, Mechanism and Machine Theory, Vol. 28, No.4 (1993), pp. 539-552.

Söylemez, E. and Freudenstein, F., Transmission optimization of spatial 4-link mechanisms, Mechanism and Machine Theory, Vol. 17, No. 4 (1982), pp. 263-283.

Tanık, E., Transmission angle in compliant slider-crank mechanism, Mechanism and Machine Theory, Vol. 46 (2011), pp. $1623-1632$.

Tanık, E. and Söylemez, E., Analysis and design of a compliant variable stroke mechanism, Mechanism and Machine Theory, Vol. 45 (2010), pp. 1385-1394.

Tanık, E. and Parlaktaş V., Compliant cardan universal joint, Transactions of the ASME, Journal of Mechanical Design, Vol. 134 (2012), 021011.

Tanık, E. and Parlaktaş, V., A new type of compliant spatial four-bar (RSSR) mechanism, Mechanism and Machine Theory, Vol. 46 (2011), pp. 593-606.

Wang, B., Zhou, L. Z., J1, C. D., Hu, H. D. and Chen, X. B., Optimal design and analysis of cross hinge four-bar mechanism based on spatial switching method, Journal of Advanced Mechanical Design, Systems, and Manufacturing, Vol. 7, No. 3 (2013), pp. 305-316.

Zhang, J., Reliability analysis of spherical function generating mechanisms , Journal of Advanced Mechanical Design, Systems, and Manufacturing, Vol. 8, No. 1 (2014), JAMDSM0007. 